Mathematical Modelling AND AnAlysis

Volume 19 Number 4, September 2014, 568-588

http://dx.doi.org/10.3846/13926292.2014.963718

(C) Vilnius Gediminas Technical University, 2014
Publisher: Taylor\&Francis and VGTU

http://www.tandfonline.com/TMMA

Print ISSN: 1392-6292

Online ISSN: 1648-3510

\title{
On Mathematical Modelling of Metals Distribution in Peat Layers
}

\section{Ilmārs Kangro ${ }^{a}$, Harijs Kalis ${ }^{b}$, Aigars Gedroics ${ }^{b}$, $\bar{E}$ rika Teirumnieka ${ }^{a}$ and Edmunds Teirumnieks ${ }^{a}$}

\author{
${ }^{a}$ Rēzekne Higher Education Institution, Department of Engineering Science \\ Atbrïivoŝanas aleja 90, LV-4601 Rēzekne, Latvia \\ ${ }^{b}$ Faculty of Physics and Mathematics, University of Latvia \\ Zellu ielā 8, LV-1002 Rīga, Latvia \\ E-mail(corresp.): kangro@ru.1v \\ E-mail: kalis@lanet.lv \\ E-mail: aigars.gedroics@lu.lv \\ E-mail: erika.teirumnieka@ru.lv \\ E-mail: edmunds.teirumnieks@ru.lv
}

Received October 22, 2013; revised September 3, 2014; published online September 10, 2014

\begin{abstract}
In this paper we consider averaging and finite difference methods for solving the 3-D boundary-value problem in multilayered domain. We consider the metals $\mathbf{F e}$ and $\mathbf{C a}$ concentration in the layered peat blocks. Using experimental data the mathematical model for calculation of concentration of metals in different points in peat layers is developed. A specific feature of these problems is that it is necessary to solve the 3-D boundary-value problems for elliptic type partial differential equations (PDEs) of second order with piece-wise diffusion coefficients in the layered domain. We develop here a finite-difference method for solving of a problem of one, two and three peat blocks with periodical boundary condition in $\mathrm{x}$ direction. This procedure allows to reduce the 3 -D problem to a system of $2-\mathrm{D}$ problems by using circulant matrix.
\end{abstract}

Keywords: 3-D boundary-value problem, averaging method, finite difference method, heavy metals $\mathrm{Fe}$ and $\mathrm{Ca}$, peat bog.

AMS Subject Classification: 65N06; 65N08; 34C29; 35R05; $60 \mathrm{~J} 60$.

\section{Introduction}

Peat is a mixture of plant remains in different stages of decay consisting in five main groups of organic compounds: proteins, lipids, hydrocarbons, pigments and lignin [11].

Regional climate, the nature of the vegetation, water $\mathrm{pH}$, and degree of metamorphosis may affect the characteristics of the peat [1]. 
Trace elements accumulated in peat lands have two main natural sources [11]: 1) Atmospheric deposition of soil dusts and aerosols; 2) The incorporation as particulate matter or in solution via runoff and ground waters (by mineral dissolution or desorption of compounds previously accumulated in the environment). Main anthropogenic pollution sources are atmospheric particles, waste waters, results of changes in environmental conditions such as changes in $\mathrm{pH}$ value [11]. Although at trace levels some heavy metals are essential for plants and animals, at higher concentrations they become dangerous for any form of life $[10]$.

Mathematical models which based on real measurements (which were made more easily accessible locations, for example, on the earth surface, or between layers of peat and with the minimum required number of measurements) are more reasonable to use for practical estimation of necessary elements of other required points of peat layers.

The task of sufficient accuracy numerical simulation of quickly solution 3-D problems for mathematical physics in multilayered media is important in known areas of the applied sciences. To achieve this goal we consider two methods: special finite difference scheme and averaging method with integral parabolic splines. For engineering calculation the concentration of metal in peat layered block the averaging method is chosen. The finite-difference method is used only for solving the obtained 2-D problems.

A. Buikis ( [4], [5] 1994) consider different assumptions for averaging methods along the vertical coordinate. These methods were applied for the mathematical simulation of the mass transfer process in multilayered underground systems. It is necessity to solve the $3-\mathrm{D}$ initial-boundary-value problems for parabolic type partial differential equations of second order with piece-wise parameters in multilayer domain. The special spline, which interpolated middle integral values of piece-wise smooth function, is defined. With the help of this spline is reduce the $3-\mathrm{D}$ problem of mathematical physics with piece-wise coefficients to 2-D problems for system of equations.

A. Buikis and H. Kalis averaged the hydrodynamical functions (velocity, stream function, temperature) in the z-direction by mathematical simulation of an electrolytic cell for aluminum production (two layers). For the viscous incompressible liquid the system of Navier-Stokes equations is considered in layered media. The equations are averaged along the height of the layers and square-law approximation of functions along z-coordinate is used [3]. The system of averaged 2-D equations is discretized on general irregular meshes and special monotone finite-difference schemes are used [8].

H. Kalis ([9] 1997) developed an effective finite-difference method for solving a problem of the above type. This method may be considered as a generalization of the method of finite volumes [12] for layered systems. This procedure allows to reduce the $3-\mathrm{D}$ problem to a system of $2-\mathrm{D}$ problem and $2-\mathrm{D}$ problems to a system of 1-D problems. The approximation is based on the conservation law approach.

A. Buikis and H. Kalis ([6] 2001) discussed the problem of radiative heating of thin plate based on the finite volume and conservative averaging methods. The aim of this paper is to verify exactness of the averaging method applied to 
the heat transfer with nonlinear Stefan-Boltzmann boundary conditions. The method of conservative averaging (AV method) is compared with the finite volumes method $(\mathrm{FV})$. These methods allow for reduction of the nonlinear 2-D heat transport problem, described by a partial differential equation, to an initial value problem stated as a system of two nonlinear ordinary differential equations (ODEs) of the first order in the time or to an initial-value problem as a system of one such equation of the first order and two nonlinear algebraic equations.

The solution of the stiff systems of ODEs have be obtained using MAPLE routines. The approximate values of temperature found by FV and AV methods are compared with values of the analytical method obtained by the Laplace transform.

M. Buike and A. Buikis ( [2] 2007) investigated the approximation error of the interpolation by integral parabolic spline $S(x)$ for the function $U(x)$, $x \in[a, b]$ itself, its first and second derivatives (here is assumed, that second and third derivatives of the function are piece-wise continuous).

They have following estimate:

$$
\left|U^{p}(x)-S^{p}(x)\right| \leq C_{p} \alpha_{N}\left\|\tilde{\Delta}_{N}\right\|^{2-p}, \quad p=0,1,2,
$$

where $\alpha_{N}=\omega\left(U^{\prime \prime},\left\|\tilde{\Delta}_{N}\right\|\right)$, Here $\omega$ is the continuity modulus for second derivatives $U^{\prime \prime}$ of interpolation function $U$ on the grid $\tilde{\Delta}_{N}: \omega(U, \delta)=\max \mid U(x+$ $h)-U(x)|| h \mid, \leq \delta, x, x+h \in[a, b]$.

\section{A Mathematical Model}

The process of diffusion the metal in the peat block is consider in 3-D parallelepiped

$$
\Omega=\{(x, y, z): 0 \leq x \leq l, 0 \leq y \leq L, 0 \leq z \leq Z\} .
$$

The domain $\Omega$ consist of multilayer medium. We will consider the stationary 3 -D problem of the linear diffusion theory for multilayered piece-wise homogeneous materials of $N$ layers in the form

$$
\Omega_{i}=\left\{(x, y, z): x \in(0, l), y \in(0, L), z \in\left(z_{i-1}, z_{i}\right)\right\}, \quad i=\overline{1, N},
$$

where $H_{i}=z_{i}-z_{i-1}$ is the height of layer $\Omega_{i}, z_{0}=0, z_{N}=Z$. We will find the distribution of concentrations $c_{i}=c_{i}(x, y, z)$ in every layer $\Omega_{i}$ at the point $(x, y, z) \in \Omega_{i}$ by solving the following partial differential equation (PDE):

$$
D_{i x} \partial^{2} c_{i} / \partial x^{2}+D_{i y} \partial^{2} c_{i} / \partial y^{2}+D_{i z} \partial^{2} c_{i} / \partial z^{2}+f_{i}(x, y, z)=0,
$$

where $D_{i x}, D_{i y}, D_{i z}$, are constant diffusion coefficients, $c_{i}=c_{i}(x, y, z)-$ the concentrations functions in every layer, $f_{i}(x, y, z)$ - the fixed sours function.

The values $c_{i}$ and the flux functions $D_{i z} \partial c_{i} / \partial z$ must be continues on the contact lines between the layers $z=z_{i}, i=\overline{1, N-1}$ :

$$
\begin{aligned}
& \left.c_{i}\right|_{z_{i}}=\left.c_{i+1}\right|_{z_{i}}, \\
& D_{i z} \partial c_{i} /\left.\partial z\right|_{z_{i}}=D_{(i+1) z} \partial c_{i+1} /\left.\partial z\right|_{z_{i}},
\end{aligned}
$$


where $i=\overline{1, N-1}$.

We assume that the layered material is bounded above and below with the plane surfaces $z=0, z=Z$ with fixed boundary conditions in following form:

$$
D_{1 z} \frac{\partial c_{1}(x, y, 0)}{\partial z}-\alpha\left(c_{1}(x, y, 0)-C_{0}(x, y)\right)=0, \quad c_{N}(x, y, Z)=C_{a}(x, y),
$$

where $C_{0}, C_{a}$ are given concentration-functions, $\alpha$ is the mass transfer coefficient. We have two form of fixed boundary conditions in the $x, y$ directions:

1) the periodical conditions by $x=0, x=l$ in the form

$$
c_{i}(0, y, z)=c_{i}(l, y, z), \quad \partial c_{i}(0, y, z) / \partial x=\partial c_{i}(l, y, z) / \partial x
$$

2) the symmetrical conditions by $y=0, y=L$

$$
\partial c_{i}(x, 0, z) / \partial y=\partial c_{i}(x, L, z) / \partial y=0 .
$$

For solving the problem (2.1)-(2.3) we will consider conservative averaging $(\mathrm{AV})$ and finite difference (FD) methods. These procedures allow to reduce the 3 -D problem to 2D boundary value problem for the system of partial differential equations with circulant matrix in the $x$-directions.

\section{The AV-Method with Quadratic Splines}

The equation of (2.1) are averaged along the heights $H_{i}$ of the layers $\Omega_{i}$ and quadratic integral splines along $z$ coordinate in following form one used $[2,4,7]$

$$
c_{i}(x, y, z)=C_{i}(x, y)+m_{i}(x, y)\left(z-\overline{z_{i}}\right)+e_{i}(x, y) G_{i}\left(\left(z-\overline{z_{i}}\right)^{2} / H_{i}^{2}-1 / 12\right),
$$

where $G_{i}=H_{i} / D_{i z}, \overline{z_{i}}=\left(z_{i-1}+z_{i}\right) / 2, z \in\left[z_{i-1}, z_{i}\right], m_{i}, e_{i}, C_{i}$ are the unknown coefficients of the spline-function, $C_{i}(x, y)=H_{i}^{-1} \int_{z_{i-1}}^{z_{i}} c_{i}(x, y, z) d z$ are the average values of $c_{i}, i=\overline{1, N}$.

After averaging the system (2.1) along every layer $\Omega_{i}$, we obtain $N$ system of 2-D PDE

$$
D_{i x} \partial^{2} C_{i} / \partial x^{2}+D_{i y} \partial^{2} C_{i} / \partial y^{2}+2 H_{i}^{-1} e_{i}+F_{i}(x, y)=0,
$$

where $F_{i}=H_{i}^{-1} \int_{z_{i-1}}^{z_{i}} f_{i}(x, y, z) d z$ are the average values of $f_{i}, i=\overline{1, N}$.

From boundary conditions (2.3) follows

$$
\begin{aligned}
& \frac{6}{\alpha}\left(D_{1 z} m_{1}-e_{1}\right)+3 m_{1} H_{1}=6\left(C_{1}-C_{0}\right)+e_{1} G_{1}, \\
& 3 m_{N} H_{N}=6\left(C_{a}-C_{N}\right)-e_{N} G_{N} .
\end{aligned}
$$

From (2.2) follows

$$
\begin{aligned}
& 3 H_{i} m_{i}+e_{i} G_{i}=6\left(C_{i+1}-C_{i}\right)-3 H_{i+1} m_{i+1}+e_{i+1} G_{i+1}, \\
& D_{i z} m_{i}+e_{i}=D_{(i+1) z} m_{i+1}-e_{i+1}, \quad i=\overline{1, N-1}
\end{aligned}
$$


Excluding $m_{i+1}$ from (3.4) we get

$3 m_{i} D_{i z}\left(G_{i}+G_{i+1}\right)+e_{i}\left(G_{i}+3 G_{i+1}\right)+2 e_{i+1} G_{i+1}=6\left(C_{i+1}-C_{i}\right), \quad i=\overline{1, N-1}$.

Replacing $i$ with $i-1$ and then excluding $m_{i-1}$ we obtain

$3 m_{i} D_{i z}\left(G_{i}+G_{i-1}\right)-e_{i}\left(G_{i}+3 G_{i-1}\right)-2 e_{i-1} G_{i-1}=6\left(C_{i}-C_{i-1}\right), \quad i=\overline{2, N}$.

For determined $e_{i}$ we exclude $m_{i}$ from $(3.5),(3.6)$

$$
\begin{aligned}
2 e_{i-1} & G_{i-1}\left(G_{i}+G_{i+1}\right)+e_{i}\left(\left(G_{i}+3 G_{i-1}\right)\left(G_{i}+G_{i+1}\right)\right. \\
& \left.+\left(G_{i}+3 G_{i+1}\right)\left(G_{i}+G_{i-1}\right)\right)+2 e_{i+1} G_{i+1}\left(G_{i}+G_{i-1}\right) \\
& =6\left(C_{i+1}-C_{i}\right)\left(G_{i}+G_{i-1}\right)-6\left(C_{i}-C_{i-1}\right)\left(G_{i}+G_{i+1}\right), \quad i=\overline{2, N-1} .
\end{aligned}
$$

From (3.5), (3.6) by $i=1, i=N$ follows

$3 m_{1} D_{1 z}\left(G_{1}+G_{2}\right)+e_{1}\left(G_{1}+3 G_{2}\right)+2 e_{2} G_{2}=6\left(C_{2}-C_{1}\right)$,

$3 m_{N} D_{N z}\left(G_{N}+G_{N-1}\right)-e_{N}\left(G_{N}+3 G_{N-1}\right)-2 e_{N-1} G_{N-1}=6\left(C_{N}-C_{N-1}\right)$.

Excluding from (3.6), (3.8) $m_{1}, m_{N}$ we obtain equations for $e_{1}, e_{N}$

$$
\begin{aligned}
& e_{1}\left[2 G_{1}+4 G_{2}+\frac{2}{\alpha}\left(4+\frac{6 G_{2}}{G_{1}}\right)\right]+2 e_{2}\left(G_{2}+\frac{2 G_{2}}{\alpha G_{1}}\right) \\
& =6\left(C_{2}-C_{1}\right)\left(1-\frac{2}{\alpha G_{1}}\right)-6\left(C_{1}-C_{0}\right)\left(1+\frac{G_{2}}{G_{1}}\right), \\
& e_{N}\left(2 G_{N}+4 G_{N-1}\right)+2 e_{N-1} G_{N-1} \\
& =-6\left(C_{N}-C_{N-1}\right)+6\left(C_{a}-C_{N}\right)\left(1+\frac{G_{N-1}}{G_{N}}\right) .
\end{aligned}
$$

The system of algebraic equations (3.7), (3.9) can be solved by Thomas algorithm for tri-diagonal matrix [12]. From (3.3), (3.6) we obtain $m_{i}, i=\overline{1, N}$ :

$$
\begin{aligned}
m_{i} & =\frac{G_{i}}{3 H_{i}\left(G_{i}+G_{i-1}\right.}\left(e_{i}\left(G_{i}+3 G_{i-1}\right)+2 e_{i-1} G_{i-1}+6\left(C_{i}-C_{i-1}\right)\right], i=\overline{2, N}, \\
m_{1} & =\frac{1}{3 H_{1}+6 D_{1 z} / \alpha}\left[6\left(C_{1}-C_{0}\right)+e_{1}\left(G_{1}+6 / \alpha\right) .\right.
\end{aligned}
$$

\subsection{The AV-method for 3, 2 and 1 layers}

In the case $N=3$ (three layers) we have equations (3.9) and (3.7) for $i=2$. Then

$$
\begin{aligned}
e_{i} & =e_{i, 1} C_{1}+e_{i, 2} C_{2}+e_{i, 3} C_{3}+e_{i, 0}, \\
m_{i} & =m_{i, 1} C_{1}+m_{i, 2} C_{2}+m_{i, 3} C_{3}+m_{i, 0}, \quad i=1 ; 2 ; 3,
\end{aligned}
$$

where

$$
e_{2,1}=\left(6\left(G_{2}+G_{3}\right)+b_{1} k_{1,1}\left(2+k_{2,1}+\alpha_{1}\right)\right) / d,
$$




$$
\begin{aligned}
& e_{2,2}=-\left(6\left(2 G_{2}+G_{3}+G_{1}\right)+b_{1} k_{1,1}\left(1+\alpha_{1}\right)+b_{3} k_{2,2}\right) / d \text {, } \\
& e_{2,3}=\left(6\left(G_{2}+G_{1}\right)+b_{3} k_{2,2}\left(2+k_{2,3}\right)\right) / d, \quad \alpha_{1}=\frac{2}{\alpha G_{1}}, \\
& e_{2,0}=-\left(b _ { 1 } k _ { 1 , 1 } \left(1+k_{2,1} C_{0}+b_{3} k_{2,2}\left(1+k_{2,3} C_{a}\right) / d, \quad d=a_{1}+a_{2}+a_{3},\right.\right. \\
& a_{2}=\left(G_{2}+3 G_{3}\right)\left(G_{2}+G_{1}\right)+\left(G_{2}+G_{3}\right)\left(G_{2}+3 G_{1}\right), \quad a_{1}=-a_{2,1} b_{1} \text {, } \\
& a_{3}=-a_{2,3} b_{3}, \quad b_{1}=2 G_{1}\left(G_{2}+G_{3}\right), \quad b_{3}=2 G_{3}\left(G_{2}+G_{1}\right), \\
& a_{2,1}=\frac{G_{2}+2 k_{2,1} / \alpha}{G_{1}+2 G_{2}+a l f}, \quad a_{2,3}=k_{2,3} /\left(1+2 k_{2,3}\right), \quad k_{2,1}=G_{2} / G_{1}, \\
& k_{2,3}=G_{2} / G_{3}, \quad k_{1,1}=3 /\left(G_{1}+2 G_{2}+\text { alf }\right), \quad k_{2,2}=3 /\left(G_{3}+2 G_{2}\right), \\
& \text { alf }=\left(4+6 k_{2,1}\right) / \alpha, \quad e_{1,1}=-a_{2,1} e_{2,1}-k_{1,1}\left(2+k_{2,1}+\alpha_{1}\right), \\
& e_{1,2}=-a_{2,1} e_{2,2}+k_{1,1}\left(1+\alpha_{1}\right), \quad e_{1,3}=-a_{2,1} e_{2,3}, \\
& e_{1,0}=-a_{2,1} e_{2,0}+k_{1,1}\left(1+k_{2,1}\right) C_{0}, \quad e_{3,1}=-a_{2,3} e_{2,1}, \\
& e_{3,2}=-a_{2,3} e_{2,2}+k_{2,2}, \quad e_{3,3}=-a_{2,3} e_{2,3}-k_{2,2}\left(2+k_{2,3}\right) \text {, } \\
& e_{3,0}=-a_{2,3} e_{2,0}+k_{2,2}\left(1+k_{2,3}\right) C a, \\
& m_{1,1}=-\left(g_{1,2} e_{1,1}+g_{3,2} e_{2,1}+6\right) / d_{1}, \quad m_{1,2}=-\left(g_{1,2} e_{1,2}+g_{3,2} e_{2,2}-6\right) / d_{1} \text {, } \\
& m_{1,3}=-\left(g_{1,2} e_{1,3}+g_{3,2} e_{2,3}\right) / d_{1}, \quad m_{1,0}=-\left(g_{1,2} e_{1,0}+g_{3,2} e_{2,0}\right) / d_{1}, \\
& d_{1}=3\left(1+k_{2,1}\right) H_{1}, \quad g_{1,2}=G_{1}+3 G_{2}, \quad g_{3,2}=2 G_{2}, \\
& m_{3,1}=\left(g_{3,3} e_{3,1}+g_{3,2} e_{2,1}\right) / d_{3}, \\
& m_{3,2}=\left(g_{3,3} e_{3,2}+g_{3,2} e_{2,2}-6\right) / d_{3}, \quad m_{3,3}=\left(g_{3,3} e_{3,3}+g_{3,2} e_{2,3}+6\right) / d_{3}, \\
& m_{3,0}=\left(g_{3,3} e_{3,0}+g_{3,2} e_{2,0}\right) / d_{3}, \quad d_{3}=3\left(1+k_{2,3}\right) H_{3}, \quad g_{3,3}=G_{3}+3 G_{2}, \\
& m_{2,1}=\left(g_{2,2} e_{2,1}+g_{2,1} e_{1,1}-6\right) / d_{2}, \quad m_{2,2}=\left(g_{2,2} e_{2,2}+g_{2,1} e_{1,2}+6\right) / d_{2}, \\
& m_{2,3}=\left(g_{2,2} e_{2,3}+g_{2,1} e_{1,3}\right) / d_{2}, \quad m_{2,0}=\left(g_{2,2} e_{2,0}+g_{2,1} e_{1,0}\right) / d_{2}, \\
& d_{2}=3 H_{2}\left(1+k_{2,1}\right) / k_{2,1}, \quad g_{2,2}=G_{2}+3 G_{1}, \quad g_{2,1}=2 G_{1},
\end{aligned}
$$

We can obtain that $e_{1,2}=e_{2,1}, e_{1,3}=e_{3,1}, e_{3,2}=e_{2,3}, e_{1,2}=e_{2,1}$, and the matrix of the 3 . order with the elements $e_{i, j}$ is symmetric and $e_{1,1}<0, e_{2,2}<0$, $e_{3,3}<0$.

From $(3.2),(3.11)$ follows the system of three PDE

$$
\left\{\begin{array}{l}
D_{1 x} \partial^{2} C_{1}(x, y) / \partial x^{2}+D_{1 y} \partial^{2} C_{1}(x, y) / \partial y^{2}+2 H_{1}^{-1} e_{1}(x, y)+\tilde{F}_{1}(x, y)=0 \\
D_{2 x} \partial^{2} C_{2}(x, y) / \partial x^{2}+D_{2 y} \partial^{2} C_{2}(x, y) / \partial y^{2}+2 H_{2}^{-1} e_{2}(x, y)+\tilde{F}_{2}(x, y)=0 \\
D_{3 x} \partial^{2} C_{3}(x, y) / \partial x^{2}+D_{3 y} \partial^{2} C_{3}(x, y) / \partial y^{2}+2 H_{3}^{-1} e_{3}(x, y)+\tilde{F}_{3}(x, y)=0
\end{array}\right.
$$

where $\tilde{F}_{i}(x, y)=F_{i}(x, y)+2 H_{1}^{-1} e_{i, 0}, i=1 ; 2 ; 3$.

In the case $N=2$ (two layers) we have coefficients from (3.11) $i=1 ; 2$

$$
\begin{gathered}
e_{1,1}=-\left(a_{2,1} k_{2,2}+k_{1,1}\left(2+k_{2,1}+\alpha_{1}\right)\right) / d \\
e_{1,2}=\left(a_{2,1} k_{2,3}+k_{1,1}\left(1+\alpha_{1}\right)\right) / d \\
e_{1,0}=\left(-a_{2,1} k_{2,4} C_{a}+k_{1,1}\left(1+k_{2,1}\right) C_{0}\right) / d, \quad k_{2,2}=3 /\left(G_{2}+2 G_{1}\right), \\
k_{2,3}=k_{2,2}\left(2+k_{1,2}\right), \quad k_{2,4}=k_{2,2}\left(1+k_{1,2}\right), \quad d=1-a_{1,3}, \\
a_{1,3}=a_{2,1} a_{2,3}, \quad a_{2,3}=1 /\left(2+k_{2,1}\right), \quad e_{2,1}=\left(-a_{2,3} e_{1,1}+k_{2,2},\right.
\end{gathered}
$$




$$
\begin{gathered}
e_{2,2}=-a_{2,3} e_{1,2}-k_{2,3}, \quad e_{2,0}=-a_{2,3} e_{1,0}-k_{2,4} C_{a}, \\
m_{1,1}=\left(g_{1,1} e_{1,1}+6\right) / d_{1}, \quad m_{1,2}=g_{1,1} e_{1,2} / d_{1}, \\
m_{1,0}=\left(g_{1,1} e_{1,0}-6 C_{0}\right) / d_{1}, \quad d_{1}=3 H_{1}+6 D_{1 z} / \alpha, \quad m_{2,1}=-G_{2} e_{2,1} / d_{2}, \\
m_{2,2}=-\left(G_{2} e_{2,1}+6\right) / d_{2}, \quad m_{2,0}=\left(-G_{2} e_{2,0}+6 C_{a}\right) / d_{2}, \quad d_{2}=3 H_{2} .
\end{gathered}
$$

From (3.12) follows the system of two PDE.

In the case $N=1$ (one layer) we have coefficients from (3.11) $i=1$

$$
\begin{aligned}
& e_{1,1}=-a_{1,1}\left(2 G_{1}+2 / \alpha\right), \quad e_{1,0}=a_{1,1}\left(G_{1} C_{0}+\left(G_{1}+2 / \alpha\right) C_{a}\right), \\
& a_{1,1}=\frac{3}{G_{1}\left(G_{1}+4 / \alpha\right)}, \quad m_{1,1}=-\left(G_{1} e_{1,1}+6\right) / d \\
& m_{1,0}=-\left(G_{1} e_{1,0}-6 C_{a}\right) / d, \quad d=3 G_{1} .
\end{aligned}
$$

From (3.12) one PDE follows.

\section{The Finite Difference Method}

For solving 2-D problems we consider an uniform grid $\left(N_{x} \times\left(N_{y}+1\right)\right): \omega_{h}=$ $\left\{\left(x_{i}, y_{j}\right), x_{i}=i h_{x}, y_{j}=(j-1) h_{y}, i=\overline{1, N_{x}}, j=\overline{1, N_{y}+1}, N_{x} h_{x}=l\right.$, $\left.N_{y} h_{y}=L\right\}$. Subscripts $(i, j)$ refer to $x, y$ indices, the mesh spacing in the $x_{i}$, $y_{j}$ directions are $h_{x}$ and $h_{y}$.

We can the PDEs (3.12) rewritten in following vector form:

$$
D_{x} \partial^{2} C / \partial x^{2}+D_{y} \partial^{2} C / \partial y^{2}-A C+\tilde{F}=0,
$$

where $D_{x}, D_{y}$ are the 3 order diagonal matrices with elements $D_{1 x}, D_{2 x}, D_{3 x}$ and $D_{1 y}, D_{2 y}, D_{3 y}, C$ is the 3 order vectors-column with elements $C_{1}, C_{2}$, $C_{3}, \tilde{F}$ is also the vectors-column with elements $\tilde{F}_{1}, \tilde{F}_{2}, \tilde{F}_{3}$, and $A$ is the block matrix in following form:

$$
A=-2\left(\begin{array}{lll}
e_{1,1} / H_{1} & e_{1,2} / H_{1} & e_{1,3} / H_{1} \\
e_{2,1} / H_{2} & e_{2,2} / H_{2} & e_{2,3} / H_{2} \\
e_{3,1} / H_{3} & e_{3,2} / H_{3} & e_{3,3} / H_{3}
\end{array}\right)
$$

The matrix A has positive eigenvalues and the system (4.1) is correctly focused.

For 2 and 1 layer we have the matrix with 2 and 1 blocks.

The equation (4.1) with periodical conditions for vector function $C$ in the uniform grid $\left(x_{i}, y_{j}\right)$ is replaced by vector difference equations of second order approximation:

$$
A A W_{j-1}-C C W_{j}+B B W_{j+1}+\bar{F}_{j}=0,
$$

where $W_{j}, \bar{F}_{j}, j=\overline{2, N_{y}}$ are the $M \times N,\left(M=N_{x}\right)$ order vectors-column with elements $C_{k, i, j} \approx C_{k}\left(x_{i}, y_{j}\right), \bar{F}_{k, i, j}=\tilde{F}_{k}\left(x_{i}, y_{j}\right), i=\overline{1, M}, k=1 ; 2 ; 3$, $A A, C C, B B=A A$ are the 3 block-matrices of $M$ order circulant symmetric matrix [13]. The circulant matrix can to give with the first rows and the calculation (matrix inversion and multiplication) can be carried out with MATLAB using simple formulae for obtaining the first $M$ elements of matrix. 
The boundary conditions are replaced by difference equations of first order approximation:

$$
C\left(x, h_{y}\right)=C(x, 0)+O\left(h_{y}\right), \quad C(x, L)=C\left(x, L-h_{y}\right)+O\left(h_{y}\right)
$$

The vectors-column $W_{j}$ from (4.2) is calculated by Thomas algorithm [12] in the matrix form using MATLAB.

$$
W_{j}=X_{j} W_{j+1}+Y_{j}=0, \quad j=N_{y}(-1) 1,
$$

where $X_{j}, Y_{j}$ are corresponding matrices and vectors, obtaining of following expressions

$$
\begin{aligned}
X_{j} & =\left(C C_{j}-A A_{j} X_{j-1}\right)^{-1} B B_{j}, \\
Y_{j} & =\left(C C_{j}-A A_{j} X_{j-1}\right)^{-1}\left(A A_{j} Y_{j}+F_{j}\right), \quad j=2(1) N_{y} .
\end{aligned}
$$

Here $X_{1}=E, Y_{1}=0, W_{\bar{N}+1}=\left(E-X_{\bar{N}}\right)^{-1} Y_{\bar{N}},\left(\bar{N}=N_{y}\right)$ where $E$ is unit matrix.

Solving 3D problems in comparing with the standard FDS methods we use circulant matrices in one direction thus the periodical boundary conditions are approximated. This allows saving CPU time by using Thomas algorithm. However, analytical modifications become more complicated in the case of 3 layers due to the calculations with 3rd order block-matrices, which elements are circulant matrices. But in the case of $N>3$ the algorithm's building become more complicated. To perform calculations in the case of multiple layers $(N>3)$ being based on the following approach is complicate. Then it is better to use well-known finite difference techniques with alternating direction methods, by reducing $3 \mathrm{D}$ problems on $1 \mathrm{D}$ due to iterative procedures.

\section{Approbation of Numerical Algorithms}

We consider following test for the approbation of the calculations: $f_{1}=f_{2}=$ $f_{3}=C_{0}=0, \alpha=6 D_{1 z} ; 600 D_{1 z} . C_{a}=C 0_{a} \cos (\pi y / L) \sin (2 \pi x / l), C 0_{a}=1$.

\section{$5.1 \quad$ Three layers}

The special solutions in the form $c_{i}(x, y, z)=g_{i}(z) \cos (\pi y / L) \sin (2 \pi x / l), i=$ $1 ; 2 ; 3$ of the PDE (2.1) can be obtain from following boundary value problem for three ODE (for conditions (2.2), (2.3));

$$
\begin{aligned}
& g_{1}^{\prime \prime}(z)-a_{1}^{2} g_{1}(z)=0, \quad D_{1 z} g_{1}^{\prime}(0)-\alpha\left(g_{1}(0)-C_{0}\right)=0, \\
& g_{2}^{\prime \prime}(z)-a_{2}^{2} g_{2}(z)=0, \quad g_{3}^{\prime \prime}(z)-a_{3}^{2} g_{3}(z)=0, \\
& g_{3}(Z)=C 0_{a}, \quad g_{1}\left(H_{1}\right)=g_{2}\left(H_{1}\right), \quad D_{1 z} g_{1}^{\prime}\left(H_{1}\right)=D_{2 z} g_{2}^{\prime}\left(H_{1}\right), \\
& g_{2}\left(L_{1}\right)=g_{3}\left(L_{1}\right), \quad D_{2 z} g_{2}^{\prime}\left(L_{1}\right)=D_{3 z} g_{3}^{\prime}\left(L_{1}\right),
\end{aligned}
$$

where $L_{1}=H_{1}+H_{2}, a_{i}=\pi \sqrt{\left(\frac{4 D_{i x}}{l^{2}}+\frac{D_{i y}}{L^{2}}\right) / D_{i z}}, i=1 ; 2 ; 3$. 
Table 1. The analytical and numerical results for 3 layers (max. and min. values \pm ).

\begin{tabular}{lrrrl}
\hline & $\alpha=600$ & $\alpha=600$ & $\alpha=6$ & $\alpha=6$ \\
\hline$z_{k}$ & $c_{a p}$ & $c_{a n}$ & $c_{a p}$ & $c_{a n}$ \\
\hline 0.00 & .0020 & .0021 & .1206 & .1257 \\
0.25 & .0328 & .0339 & .1395 & .1451 \\
0.50 & .0642 & .0659 & .1599 & .1659 \\
0.75 & .0963 & .0986 & .1820 & .1883 \\
1.00 & .1292 & .1321 & .2057 & .2123 \\
1.25 & .1476 & .1508 & .2196 & .2264 \\
1.50 & .1671 & .1705 & .2350 & .2420 \\
1.75 & .1877 & .1912 & .2521 & .2591 \\
2.00 & .2096 & .2133 & .2709 & .2780 \\
2.25 & .2331 & .2367 & .2917 & .2986 \\
2.50 & .2578 & .2617 & .3142 & .3213 \\
2.75 & .6189 & .6272 & .6469 & .6568 \\
\hline
\end{tabular}

Therefore the exact solution is

$$
\begin{aligned}
& g_{1}(z)=P_{1} \sinh \left(a_{1} z\right)+P_{0} \cosh \left(a_{1} z\right), \quad g_{2}(z)=P_{2} \sinh \left(a_{2} z\right)+P_{3} \cosh \left(a_{2} z\right), \\
& g_{3}(z)=P_{4} \sinh \left(a_{3} z\right)+P_{5} \cosh \left(a_{3} z\right),
\end{aligned}
$$

where the constants $\overline{P_{0}, P_{5}}$ are calculated from (5.1).

The averaged values are

$$
\begin{aligned}
H_{1}^{-1} \int_{0}^{H_{1}} g_{1}(z) d z= & \frac{1}{H_{1} a_{1}}\left(P_{1}\left(\cosh \left(a_{1} H_{1}\right)-1\right)+P_{0} \sinh \left(a_{1} H_{1}\right)\right) \\
H_{2}^{-1} \int_{H_{1}}^{L_{1}} g_{2}(z) d z= & \frac{1}{H_{2} a_{2}}\left(P_{3}\left(\sinh \left(a_{2} L_{1}\right)-\sinh \left(a_{2} H_{1}\right)\right)\right. \\
& \left.+P_{2}\left(\cosh \left(a_{2} L_{1}\right)-\cosh \left(a_{2} H_{1}\right)\right)\right) \\
H_{3}^{-1} \int_{L_{1}}^{Z} g_{3}(z) d z= & \frac{1}{H_{3} a_{3}}\left(P_{5}\left(\sinh \left(a_{3} Z\right)-\sinh \left(a_{3} L_{1}\right)\right)\right. \\
& \left.+P_{4}\left(\cosh \left(a_{3} Z\right)-\cosh \left(a_{3} L_{1}\right)\right)\right) .
\end{aligned}
$$

We have the following numerical results $\left(H_{1}=1, H_{2}=1.5, H_{3}=0.5, Z=3.0\right.$, $\left.C_{0}=0, C 0_{a}=1\right)$ for maximal and minimal values of $c_{k}$ in the plane $z=z_{k}$, $z_{k}=(k-1) h_{z}, k=\overline{1,12}, z_{13}=Z, z_{5}=H_{1}, z_{11}=H_{1}+H_{2}, h_{z}=0.25$ by:

$$
\begin{aligned}
& D_{1 z}=10^{-3}, D_{2 z}=1.87510^{-3}, D_{3 z}=0.133310^{-3}, \alpha=600 D_{1 z}, \alpha=6 D_{1 z}, \\
& D_{1 x}=D_{1 y}=310^{-4}, \quad D_{2 x}=D_{2 y}=410^{-4}, \quad D_{3 x}=D_{3 y}=510^{-5} .
\end{aligned}
$$

The numerical results by $N_{x}=N_{y}=20, N_{z}=12$ is given in Table $1\left(c_{a p}\right.$, $c_{a n}$ are the approximate and analytical-exact values. We have the following averaged (integral) values:

1) $\alpha=600 D_{1 z}-$ for $C_{a p}: C_{1}=0.0646, C_{2}=0.1894, C_{3}=0.6202$; for $C_{a n}$ : $C_{1}=0.0663, C_{2}=0.1931, C_{3}=0.6284$; 
2) $\alpha=6 D_{1 z}$ - for $C_{a p}: C_{1}=0.1609, C_{2}=0.2545, C_{3}=0.6482$; for $C_{a n}$ : $C_{1}=0.1669, C_{2}=0.2617, C_{3}=0.6581$.

The numerical results for $N_{x}=N_{y}=40$ are coincided with 3 decimal places.

\subsection{Two layers}

The special solutions in the form $c_{i}(x, y, z)=g_{i}(z) \cos (\pi y / L) \sin (2 \pi x / l), i=$ $1 ; 2$ of the PDE (2.1) can be obtain from following boundary value problem for two ODE (for conditions $(2.2),(2.3)$ ):

$$
\begin{gathered}
g_{1}^{\prime \prime}(z)-a_{1}^{2} g_{1}(z)=0, \quad D_{1 z} g_{1}^{\prime}(0)-\alpha\left(g_{1}(0)-C_{0}\right)=0, \\
g_{2}^{\prime \prime}(z)-a_{2}^{2} g_{2}(z)=0, \quad g_{2}(Z)=C 0_{a}, \\
g_{1}\left(H_{1}\right)=g_{2}\left(H_{1}\right), \quad D_{1 z} g_{1}^{\prime}\left(H_{1}\right)=D_{2 z} g_{2}^{\prime}\left(H_{1}\right),
\end{gathered}
$$

where $Z=H_{1}+H_{2}, a_{i}=\pi \sqrt{\left(\frac{4 D_{i x}}{l^{2}}+\frac{D_{i y}}{L^{2}}\right) / D_{i z}}, i=1 ; 2$.

Therefore the exact solution is

$$
g_{1}(z)=P_{1} \sinh \left(a_{1} z\right)+P_{0} \cosh \left(a_{1} z\right), \quad g_{2}(z)=P_{2} \sinh \left(a_{2} z\right)+P_{3} \cosh \left(a_{2} z\right),
$$

where the constants $\overline{P_{0}, P_{3}}$ are calculated from (5.2).

The averaged values are

$$
\begin{aligned}
H_{1}^{-1} \int_{0}^{H_{1}} g_{1}(z) d z= & \frac{1}{H_{1} a_{1}}\left(P_{1}\left(\cosh \left(a_{1} H_{1}\right)-1\right)+P_{0} \sinh \left(a_{1} H_{1}\right)\right) \\
H_{2}^{-1} \int_{H_{1}}^{L_{1}} g_{2}(z) d z= & \frac{1}{H_{2} a_{2}}\left(P_{3}\left(\sinh \left(a_{2} L_{1}\right)-\sinh \left(a_{2} H_{1}\right)\right)\right. \\
& \left.+P_{2}\left(\cosh \left(a_{2} L_{1}\right)-\cosh \left(a_{2} H_{1}\right)\right)\right) .
\end{aligned}
$$

We have following numerical results $\left(H_{1}=1, H_{2}=2, Z=3.0, C_{0}=0\right.$, $C 0_{a}=1$ ) for maximal and minimal values of $c_{k}$ in the plane $z=z_{k}, z_{k}=$ $(k-1) h_{z}, k=\overline{1,12}, z_{13}=Z, z_{5}=H_{1}, z_{11}=2.5, h_{z}=0.25$ by:

$$
\begin{aligned}
& D_{1 z}=10^{-3}, \quad D_{2 z}=1.87510^{-3}, \quad \alpha=600 D_{1 z}, \quad \alpha=6 D_{1 z}, \\
& D_{1 x}=D_{1 y}=310^{-4}, \quad D_{2 x}=D_{2 y}=310^{-4} .
\end{aligned}
$$

The numerical results are given in Table 2. We have following averaged (integral) values:

1) $\alpha=600 D_{1 z}$ - for $C_{a p}: C_{1}=0.2092, C_{2}=0.6832 ;$ for $C_{a n}: C_{1}=0.2160$, $C_{2}=0.6970$;

2) $\alpha=6 D_{1 z}$ - for $C_{a p}: C_{1}=0.4481, C_{2}=0.7585$; for $C_{a n}: C_{1}=0.4647$, $C_{2}=0.7752$.

\subsection{One layer}

The special solutions in the form $c_{1}(x, y, z)=g_{1}(z) \cos (\pi y / L) \sin (2 \pi x / l), 2$ of the PDE (2.1) can be obtain in the following form: 
Table 2. The analytical and numerical results for 2 layers (max. and min. values \pm ).

\begin{tabular}{lllll}
\hline & $\alpha=600$ & $\alpha=600$ & $\alpha=6$ & $\alpha=6$ \\
\hline$z_{k}$ & $c_{a p}$ & $c_{a n}$ & $c_{a p}$ & $c_{a n}$ \\
\hline 0.00 & .0067 & .0069 & .3374 & .3498 \\
0.25 & .1072 & .1103 & .3898 & .4039 \\
0.50 & .2086 & .2147 & .4457 & .4618 \\
0.75 & .3115 & .3211 & .5064 & .5240 \\
1.00 & .4172 & .4304 & .5718 & .5911 \\
1.25 & .4759 & .4909 & .6099 & .6297 \\
1.50 & .5382 & .5537 & .6519 & .6715 \\
1.75 & .6041 & .6193 & .6979 & .7165 \\
2.00 & .6736 & .6879 & .7479 & .7651 \\
2.25 & .7468 & .7599 & .8020 & .8175 \\
2.50 & .8267 & .8357 & .8632 & .8739 \\
2.75 & .9111 & .9156 & .9292 & .9347 \\
\hline
\end{tabular}

$$
g_{1}^{\prime \prime}(z)-a_{1}^{2} g_{1}(z)=0, \quad D_{1 z} g_{1}^{\prime}(0)-\alpha\left(g_{1}(0)-C_{0}\right)=0, \quad g_{1}(Z)=C 0_{a},
$$

where $a_{1}=\pi \sqrt{\left(\frac{4 D_{1 x}}{l^{2}}+\frac{D_{1 y}}{L^{2}}\right) / D_{1 z}}$.

Therefore the exact solution is

$$
g_{1}(z)=P_{1} \sinh \left(a_{1} z\right)+P_{0} \cosh \left(a_{1} z\right),
$$

where the constants

$$
P_{1}=\frac{C 0_{a}-C_{0} \cosh \left(a_{1} Z\right)}{\sinh \left(a_{1} Z\right)+z_{1} a_{1} \cosh \left(a_{1} Z\right)}, \quad P_{0}=z_{1} a_{1} P_{1}+C_{0} .
$$

The averaged values are

$$
H_{1}^{-1} \int_{0}^{H_{1}} g_{1}(z) d z=\frac{1}{H_{1} a_{1}}\left(P_{1}\left(\cosh \left(a_{1} H_{1}\right)-1\right)+P_{0} \sinh \left(a_{1} H_{1}\right)\right) .
$$

We have following numerical results $\left(H_{1}=Z=3.0, C_{0}=0, C 0_{a}=1\right)$ for maximal and minimal values of $c_{k}$ in the plane $z=z_{k}, z_{k}=(k-1) h_{z}, k=\overline{1,12}$, $z_{13}=Z, z_{5}=1, z_{11}=2.5, h_{z}=0.25$ by:

$$
D_{1 z}=10^{-3}, \quad \alpha=600 D_{1 z}, \quad \alpha=6 D_{1 z}, \quad D_{1 x}=D_{1 y}=310^{-4} .
$$

The numerical results are given in Table 3. We have following averaged (integral) values:

1) $\alpha=600 D_{1 z}-$ for $C_{a p}: C_{1}=0.4435$; for $C_{a n}: C_{1}=0.4530$;

2) $\alpha=6 D_{1 z}-$ for $C_{a p}: C_{1}=0.5398$; for $C_{a n}: C_{1}=0.5646$.

\section{Determining the Diffusion Coefficient in the 1D Case}

After recording the diffusion coefficient in one layer and solving linear 1D problem it is possible to calculate other diffusion coefficients using experimental measurements in the contact lines between the layers. 
Table 3. The analytical and numerical results for 1 layer (max. and min. values \pm ).

\begin{tabular}{lllll}
\hline & $\alpha=600$ & $\alpha=600$ & $\alpha=6$ & $\alpha=6$ \\
\hline$z_{k}$ & $c_{a p}$ & $c_{a n}$ & $c_{a p}$ & $c_{a n}$ \\
\hline 0.00 & .0037 & .0045 & .2178 & .2518 \\
0.25 & .0618 & .0714 & .2531 & .2908 \\
0.50 & .1242 & .1390 & .2937 & .3325 \\
0.75 & .1910 & .2079 & .3395 & .3773 \\
1.00 & .2621 & .2787 & .3905 & .4256 \\
1.25 & .3376 & .3521 & .4468 & .4778 \\
1.50 & .4174 & .4288 & .5083 & .5344 \\
1.75 & .5016 & .5094 & .5751 & .5960 \\
2.00 & .5901 & .5948 & .6486 & .6631 \\
2.25 & .6853 & .6857 & .7280 & .7363 \\
2.50 & .7853 & .7829 & .8131 & .8164 \\
2.75 & .8902 & .8873 & .9038 & .9040 \\
\hline
\end{tabular}

\subsection{Three layers}

In $1 \mathrm{D}$ case we have the boundary value problem of the following 3 ODEs

$$
\begin{aligned}
& c_{1}^{\prime \prime}(z)=0, \quad c_{2}^{\prime \prime}(z)=0, \quad c_{3}^{\prime \prime}(z)=0, \\
& D_{1 z} c_{1}^{\prime}(0)-\alpha\left(c_{1}(0)-C_{0}\right)=0, \quad c_{3}(Z)=C 0_{a}, \\
& c_{1}\left(H_{1}\right)=c_{2}\left(H_{1}\right), \quad D_{1 z} c_{1}^{\prime}\left(H_{1}\right)=D_{2 z} c_{2}^{\prime}\left(H_{1}\right), \\
& c_{2}\left(L_{1}\right)=c_{3}\left(L_{1}\right), \quad D_{2 z} c_{2}^{\prime}\left(L_{1}\right)=D_{3 z} c_{3}^{\prime}\left(L_{1}\right) .
\end{aligned}
$$

Using relations $D_{1 z} / D_{2 z}=x 1, D_{1 z} / D_{3 z}=y 1, D_{1 z} / \alpha=z 1$ we have following solutions:

$$
c_{1}(z)=C_{1} z+C_{2}, \quad c_{2}(z)=C_{3} z+C_{4}, \quad c_{3}(z)=C_{5} z+C_{6},
$$

where $C_{1}=\frac{C 0_{a}-C_{0}}{H_{1}+x 1 H_{2}+y 1 H_{3}+z_{1}}, C_{2}=C_{0}+z 1 C_{1}, C_{3}=x 1 C_{1}, C_{4}=C 0_{a}-$ $C_{1}\left(L_{1} x 1+y 1 H_{3}\right), C_{5}=y 1 C_{1}, C_{6}=C 0_{a}-C_{1} y 1 Z$.

From experimentally obtained data $c_{1}(0)=C 00, c_{1}\left(H_{1}\right)=C 01, c_{2}\left(L_{1}\right)=$ $C 02$, we can determined the relations

$$
x 1=\frac{H_{1}(C 02-C 01)}{H_{2}(C 01-C 00)}, \quad y 1=\frac{H 1(C a-C 02)}{H 3(C 01-C 00)}, \quad z 1=\frac{H 1\left(C 00-C_{0}\right)}{(C 01-C 00)} .
$$

We consider the data for 2 metals in the peat blocks with $H_{1}=1, H_{2}=1.5$, $H_{3}=0.5$ :

1) for Fe: $C 0_{a}=1.88, C_{0}=0, C 00=0.66, C 01=0.83, C 02=1.50$ we get $D_{2 z}=0.38 D_{1 z}, D_{3 z}=0.22 D_{1 z}, \alpha=0.26 D_{1 z}$,

2) for Ca: $C 0_{a}=4.63, C_{0}=0, C 00=1.30, C 01=1.90, C 02=2.38$ we get $D_{2 z}=1.875 D_{1 z}, D_{3 z}=0.133 D_{1 z}, \alpha=0.46 D_{1 z}$.

We obtain with MAPLE by $D_{1 z}=10^{-3}$ Figs. 1,2 and the coefficients $C_{1}$, $C_{2}, C_{3}, C_{4}, C_{5}, C_{6}$ depending on $\mathrm{C} 00, \mathrm{C} 01, \mathrm{C} 02$. 
580 I. Kangro, H. Kalis, A. Gedroics, E. Teirumnieka and E. Teirumnieks

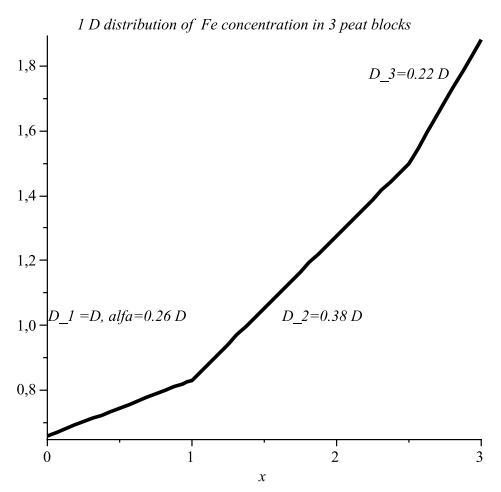

Figure 1. Fe distribution and $D_{z}$.

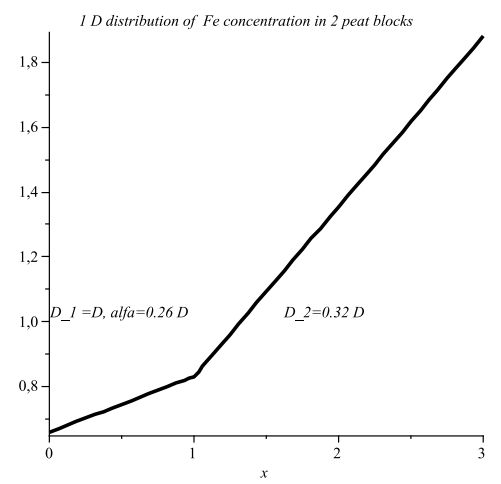

Figure 3. Fe distribution and $D_{z}$.

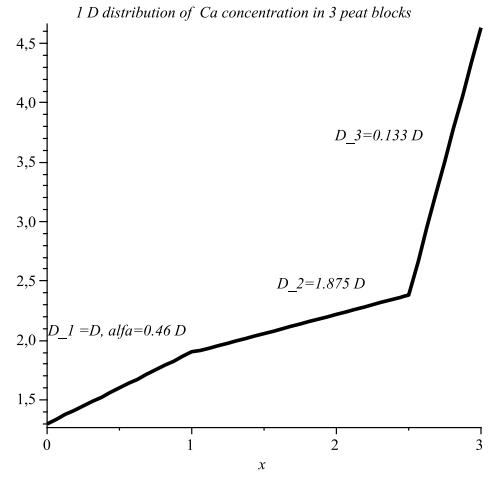

Figure 2. Ca distribution and $D_{z}$.

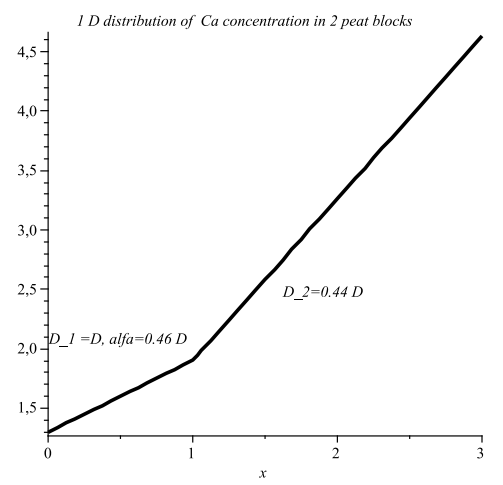

Figure 4. Ca distribution and $D_{z}$.

\subsection{Two layers}

For two layers $H_{1}=1, H_{2}=2, Z=3$ solution of ODEs (6.1) is

$$
c_{1}(z)=C_{1} z+C_{2}, \quad c_{2}(z)=C_{3} z+C_{4},
$$

where $C_{1}=\frac{C 0_{a}-C_{0}}{H_{1}+x 1 H_{2}}, C_{2}=C_{0}+z 1 C_{1}, C_{3}=x 1 C_{1}, C_{4}=C 0_{a}-C_{1} Z x 1$. From $c_{1}(0)=C 00, c_{1}\left(H_{1}\right)=C 01$ follows

$$
x 1=\frac{H_{1}\left(C 0_{a}-C 01\right)}{H_{2}(C 01-C 00)}, \quad z 1=\frac{H 1\left(C 00-C_{0}\right)}{(C 01-C 00)} .
$$

In this case for $\mathrm{Fe}-D_{2 z}=0.32 D_{1 z}, \alpha=0.26 D_{1 z}$, and for Ca- $D_{2 z}=0.44 D_{1 z}$, $\alpha=0.46 D_{1 z}$. We obtain $D_{1 z}=10^{-3}$ in Figs. 3, 4 . 


\subsection{One layer}

For one layer $H_{1}=Z=3$ solution of ODEs (6.1) is $c_{1}(z)=C_{1} z+C_{2}$, where $C_{1}=\frac{C 0_{a}-C_{0}}{H_{1}+z 1}, C_{2}=C_{0}+z 1 C_{1}$. From $c_{1}(0)=C 00$ follows

$$
z 1=\frac{H 1\left(C 00-C_{0}\right)}{\left(C 0_{a}-C 00\right)} .
$$

In this case $\left(D_{1 z}=10^{-3}\right)$ for $\mathrm{Fe}-\alpha=0.62 D_{1 z}$, and for Ca- $\alpha=0.85 D_{1 z}$.

\section{Some Numerical Results}

We consider the metals Fe and Ca concentration in the 3,2 and 1 layered peat blocks $\Omega$ (see Fig. 3) with $L=l=1 \mathrm{~m}, Z=3 . L=l=1 \mathrm{~m}, Z=$ $H_{1}+H_{2}+H_{3}=3 \mathrm{~m}, H_{1}=1 \mathrm{~m}, H_{2}=1.5 \mathrm{~m}, H_{3}=0.5 \mathrm{~m}$.

On the top of earth $(z=Z)$ are measure the concentration $c \frac{\mathrm{mg}}{\mathrm{kg}}$ of metals in following nine points in the $(x, y)$ plane:

1) for Fe:

$$
\begin{aligned}
& c(0.1,0.2)=1.69, c(0.5,0.2)=1.83, c(0.9,0.2)=1.72, c(0.1,0.5)=1.70 \\
& c(0.5,0.5)=1.88, c(0.9,0.5)=1.71, c(0.1,0.8)=1.71, c(0.5,0.8)=1.82 \\
& c(0.9,0.8)=1.73
\end{aligned}
$$

2) for $\mathrm{Ca}$ :

$$
\begin{aligned}
& c(0.1,0.2)=3.69, c(0.5,0.2)=4.43, c(0.9,0.2)=3.72, c(0.1,0.5)=4.00 \\
& c(0.5,0.5)=4.63, c(0.9,0.5)=4.11, c(0.1,0.8)=3.71, c(0.5,0.8)=4.50 \\
& c(0.9,0.8)=3.73
\end{aligned}
$$

This date are smoothing in matrix $C_{a}$ by $2 \mathrm{D}$ interpolation with MATLAB operator, using the spline function. In Figs. $7-8$ we can see the distribution of concentration $c$ for $\mathrm{Fe}$ and for $\mathrm{Ca}$ in the $(x, y)$ plane by $z=Z$.

We have examining the convergence and accuracy by reducing the mesh spacing in the direction of the $\mathrm{x}, \mathrm{y}$ twice. The vector difference equations (4.2) were solved repeatedly, reducing step $h_{x}$ and and $h_{y}$ twice and the results obtained, which coincides with 2 decimal places.

\subsection{3 layers}

For 3 layers we use $Z=H_{1}+H_{2}+H_{3}=3 m, L_{1}=H_{1}+H_{2}=2.5, H_{1}=1 \mathrm{~m}$, $H_{2}=1.5 \mathrm{~m}, H_{3}=0.5 \mathrm{~m}$ and following diffusion coefficients in the layers:

1) for $\mathrm{Fe}\left(C 00=0.66, C 0_{a}=1.88\right)$ :

$$
D_{1 z}=10^{-3}, D_{2 z}=0.38 * 10^{-3}, D_{3 z}=0.22 * 10^{-3} \text {, }
$$

2) for $\mathrm{Ca}\left(C 00=1.30, C 0_{a}=4.63\right)$ :

$$
D_{1 z}=10^{-3}, D_{2 z}=1.875 * 10^{-3}, D_{3 z}=0.1333 * 10^{-3} \text {. }
$$

The diffusion coefficients in $x, y$ directions are

$$
D_{1 x}=D_{1 y}=310^{-4}, \quad D_{2 x}=D_{2 y}=410^{-4}, \quad D_{3 x}=D_{3 y}=510^{-5} .
$$




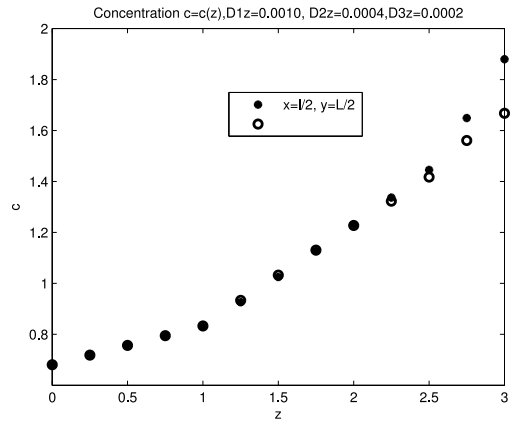

Figure 5. $c$ depending on $z$ for $\mathrm{Fe}$ in 3-layers.

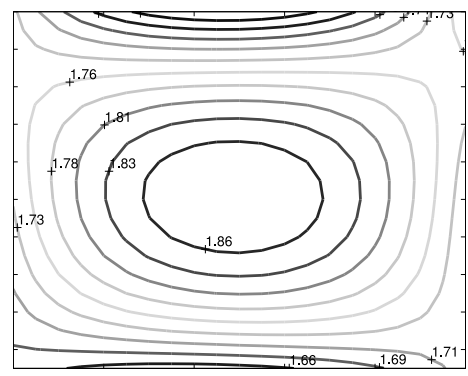

Figure 7. Levels of $c$ by $z=Z$ for Fe.

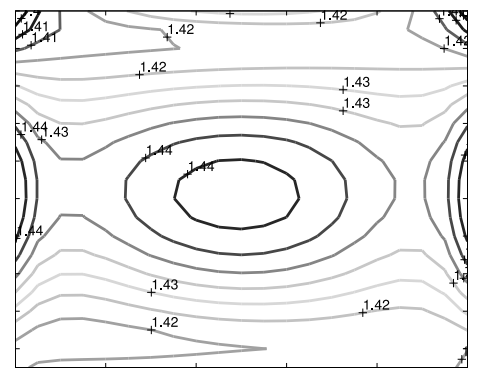

Figure 9. Levels of $c$ by $z=H_{1}+H_{2}$ for Fe in 3-layers.

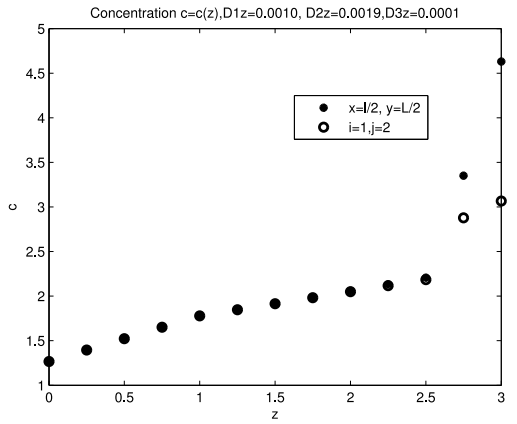

Figure 6. $c$ depending on $z$ for $\mathrm{Ca}$ in 3-layers.

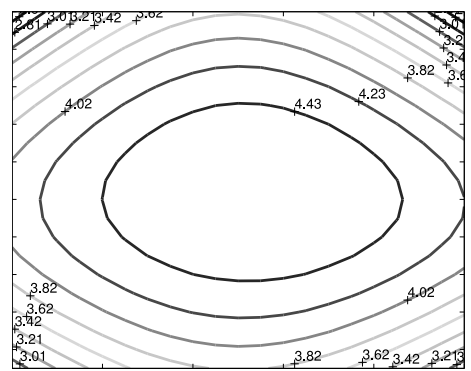

Figure 8. Levels of $c$ by $z=Z$ for Ca.

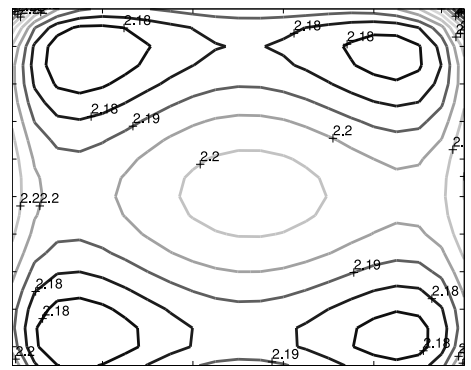

Figure 10. Levels of $c$ by $z=H_{1}+H_{2}$ for $\mathrm{Ca}$ in 3-layers.

In Figs. 5, 6 are the graphics of metals concentration $c$ depending on vertical coordinate $z$ by $x=l / 2, y=L / 2$ and in other points. In Figs. 9-12 we can see the distribution of concentration $c$ for Fe and for $\mathrm{Ca}$ in the different $(x, y)$ plane by $z=H_{1}+H_{2}, z=0$. For the peat block corresponding experimental 


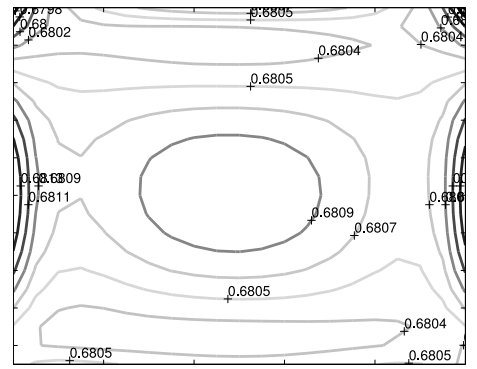

Figure 11. Levels of $c$ by $z=0$ for Fe in 3-layers.

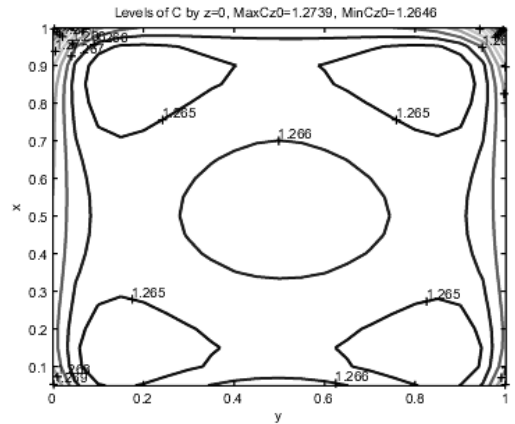

Figure 12. Levels of $c$ by $z=0$ for $\mathrm{Ca}$ in 3-layers.

Table 4. The experimental and numerical results by $x=0.5 m, y=0.5 m$ depending on $z$.

\begin{tabular}{lllll}
\hline$z$ & $c_{\text {num }}-F e$ & $c_{\text {exp }}-F e$ & $c_{\text {num }}-C a$ & $c_{\text {exp }}-C a$ \\
\hline 0.000 & 0.681 & 0.660 & 1.267 & 1.300 \\
0.250 & 0.719 & & 1.395 & \\
0.500 & 0.756 & & 1.522 & \\
0.750 & 0.793 & & 1.649 & \\
1.000 & 0.829 & 0.830 & 1.780 & 1.900 \\
1.250 & 0.926 & & 1.844 & \\
1.500 & 1.025 & & 1.914 & \\
1.750 & 1.127 & 1.150 & 1.985 & 1.980 \\
2.000 & 1.231 & & 2.057 & \\
2.250 & 1.337 & & 2.132 & \\
2.500 & 1.4458 & 1.500 & 2.207 & 2.380 \\
2.750 & 1.649 & & 3.351 & \multirow{2}{*}{4.630} \\
3.000 & 1.880 & 1.880 & 4.630 & \\
\hline
\end{tabular}

$\left(c_{\text {exp }}\right)$ and numerical $\left(c_{\text {num }}\right)$ results by $x=0.5 m, y=0.5 m$ depending on $z$ are obtained in Table 4.

\subsection{2 layers}

For 2 layers we use $Z=H_{1}+H_{2}=3 m, H_{1}=1 m, H_{2}=2 m$ and following diffusion coefficients in the layers:

1) for Fe $D_{1 z}=10^{-3}, D_{2 z}=0.38 * 10^{-3}$,

2) for Ca $D_{1 z}=10^{-3}, D_{2 z}=1.875 * 10^{-3}$.

The diffusion coefficients in $x, y$ directions are $D_{1 x}=D_{1 y}=310^{-4}, D_{2 x}=$ $D_{2 y}=410^{-4}$.

In Figs. 13, 14 are the graphics of metals concentration $c$ depending on vertical coordinate $z$ by $x=l / 2, y=L / 2$ and in other point. In Figs. 15-18 we can see the distribution of concentration $c$ for $\mathrm{Fe}$ and for $\mathrm{Ca}$ in the $(x, y)$ plane by $z=0, z=2.5$. For the peat block corresponding experimental $\left(c_{\text {exp }}\right)$ 


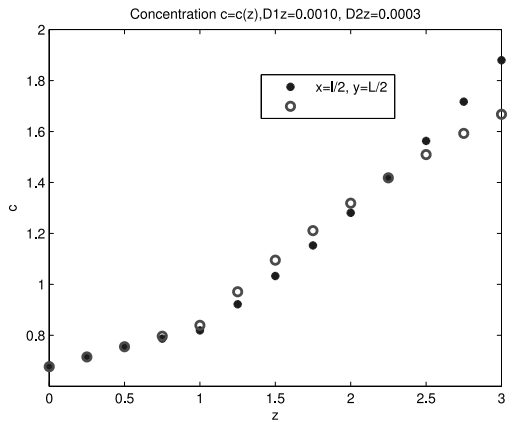

Figure 13. $c$ depending on $z$ for Fe in 2-layers.

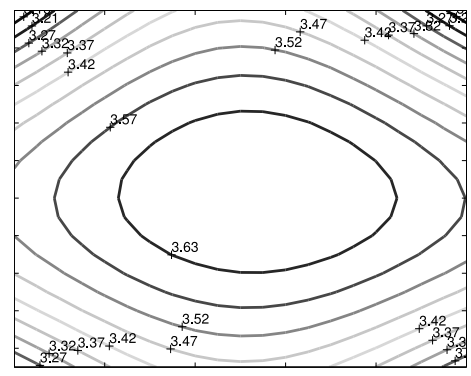

Figure 15. Levels of $c$ by $z=2.5$ for $\mathrm{Ca}$ in 2-layers.

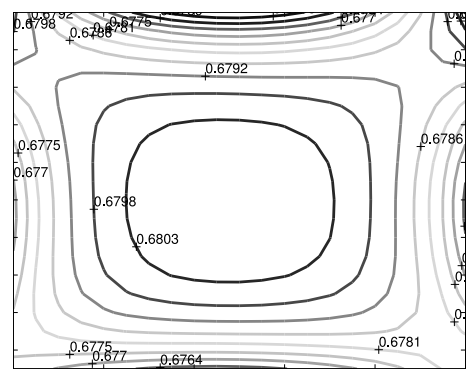

Figure 17. Levels of $c$ by $z=0$ for $\mathrm{Fe}$ in 2-layers.

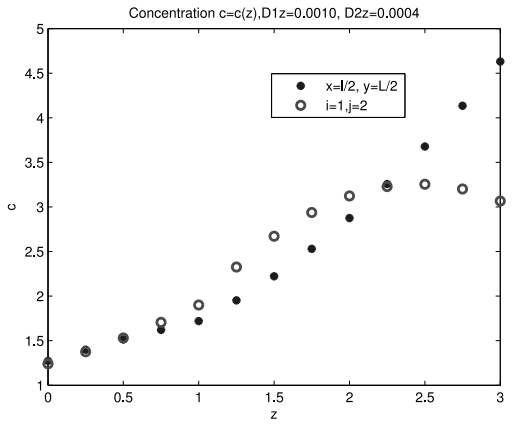

Figure 14. $c$ depending on $z$ for $\mathrm{Ca}$ in 2-layers.

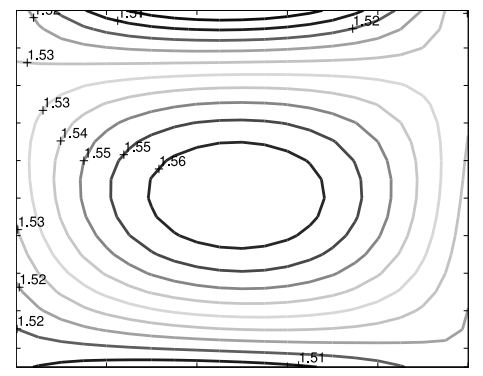

Figure 16. Levels of $c$ by $z=2.5$ for Fe in 2-layers.

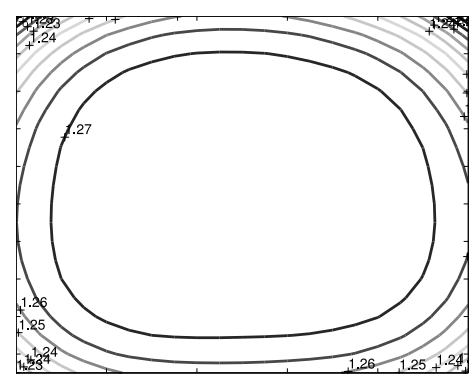

Figure 18. Levels of $c$ by $z=0$ for $\mathrm{Ca}$ in 2-layers.

and numerical $\left(c_{\text {num }}\right)$ results by $x=0.5 \mathrm{~m}, y=0.5 \mathrm{~m}$ depending on $z$ are obtained in Table 5 . 
Table 5. The experimental and numerical results by $x=0.5 m, y=0.5 m$ depending on $z$.

\begin{tabular}{lllll}
\hline$z$ & $c_{n u m}-F e$ & $c_{\text {exp }}-F e$ & $c_{n u m}-C a$ & $c_{\text {exp }}-C a$ \\
\hline 0.000 & 0.681 & 0.660 & 1.274 & 1.300 \\
0.250 & 0.718 & & 1.399 & \\
0.500 & 0.753 & & 1.515 & \\
0.750 & 0.787 & & 1.622 & \\
1.000 & 0.819 & 0.830 & 1.720 & 1.900 \\
1.250 & 0.922 & & 1.953 & \\
1.500 & 1.033 & & 2.223 & \\
1.750 & 1.153 & 1.150 & 2.530 & 1.980 \\
2.000 & 1.281 & & 2.875 & \\
2.250 & 1.418 & & 3.257 & \\
2.500 & 1.563 & 1.500 & 3.677 & 2.380 \\
2.750 & 1.717 & & 4.135 & \\
3.000 & 1.880 & 1.880 & 4.630 & 4.630 \\
\hline
\end{tabular}

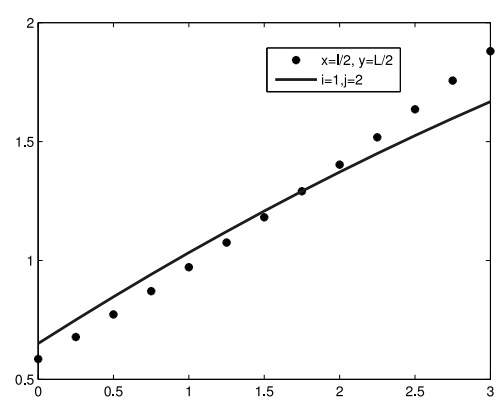

Figure 19. $c$ depending on $z$ for $\mathrm{Fe}$ in 1-layer.

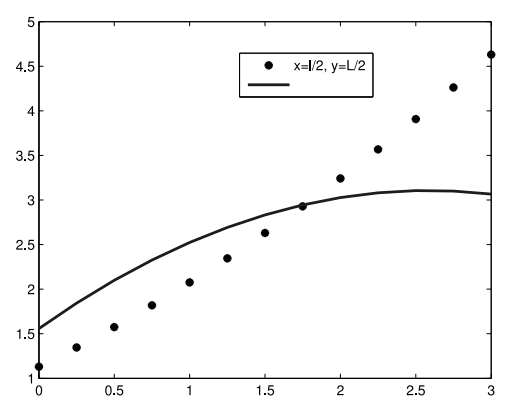

Figure 20. $c$ depending on $z$ for $\mathrm{Ca}$ in 1-layer.

\subsection{1 layer}

For one layer we use $Z=H_{1}=3 \mathrm{~m}$ and following diffusion coefficients in the layers: for $\mathrm{Fe} D_{1 z}=10^{-3}$, and for Ca also $D_{1 z}=10^{-3}$.

The diffusion coefficients in $x, y$ directions are $D_{1 x}=D_{1 y}=310^{-4}$.

In Figs. 19, 20 are the graphics of metals concentration $c$ depending on vertical coordinate $z$ by $x=l / 2, y=L / 2$ and in other point. In Figs. 21-24 we can see the distribution of concentration $c$ for Fe and for $\mathrm{Ca}$ in the $(x, y)$ plane by $z=0, z=2.5$. For the peat block corresponding experimental $\left(c_{\text {exp }}\right)$ and numerical $\left(c_{\text {num }}\right)$ results by $x=0.5 \mathrm{~m}, y=0.5 \mathrm{~m}$ depending on $z$ are obtained in Table 6 .

\section{Conclusions}

The profile of the concentration changes for metals at first may be influenced their biogenic recycling and low mobility of these elements considering also changes of water table. 


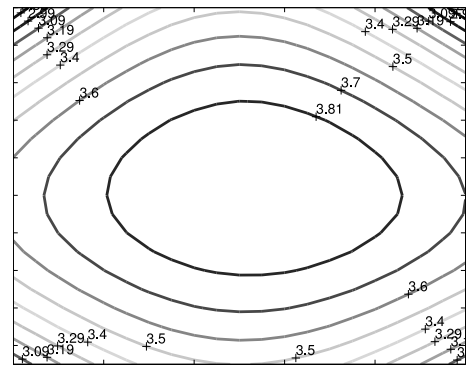

Figure 21. Levels of $c$ by $z=2.5$ for $\mathrm{Ca}$ in 1-layer.

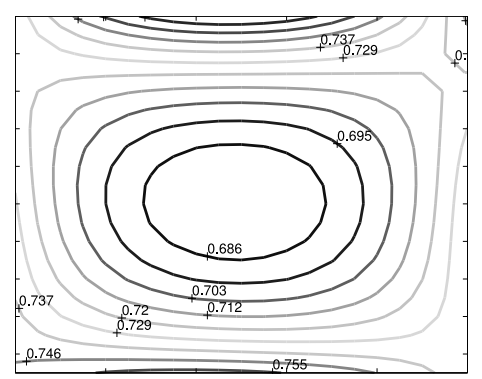

Figure 23. Levels of $c$ by $z=0$ for $\mathrm{Fe}$ in 1-layer.

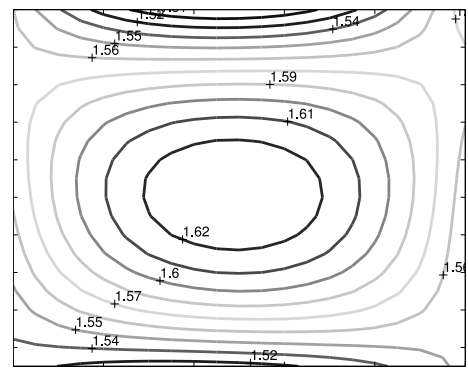

Figure 22. Levels of $c$ by $z=2.5$ for $\mathrm{Fe}$ in 1-layer.

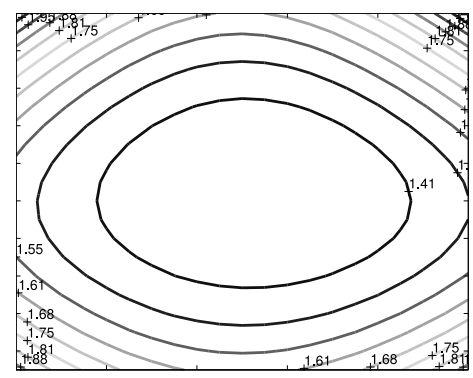

Figure 24. Levels of $c$ by $z=0$ for $\mathrm{Ca}$ in 1-layer.

Table 6. The experimental and numerical results by $x=0.5 \mathrm{~m}, y=0.5 \mathrm{~m}$ depending on $z$.

\begin{tabular}{lllll}
\hline$z$ & $c_{\text {num }}-F e$ & $c_{\text {exp }}-F e$ & $c_{\text {num }}-C a$ & $c_{\text {exp }}-C a$ \\
\hline 0.000 & 0.586 & 0.660 & 1.131 & 1.300 \\
0.250 & 0.678 & & 1.346 & \\
0.500 & 0.773 & & 1.575 & \\
0.750 & 0.871 & & 1.818 & \\
1.000 & 0.971 & 0.830 & 2.075 & 1.900 \\
1.250 & 1.075 & & 2.346 & \\
1.500 & 1.182 & & 2.631 & \\
1.750 & 1.291 & 1.150 & 2.929 & 1.980 \\
2.000 & 1.403 & & 3.242 & \\
2.250 & 1.518 & & 3.568 & \\
2.500 & 1.636 & 1.500 & 3.908 & 2.380 \\
2.750 & 1.756 & & 4.2621 & \\
3.000 & 1.880 & 1.880 & 4.630 & 4.630 \\
\hline
\end{tabular}

Changes of concentrations of studied elements in all points have similar characters - concentrations very fast decreases with depth increasing. The 
major concentrations of heavy metals are observed at the top layers of peat. Concentrations of $\mathrm{Ca}$ and Fe very fast decreases with depth increasing. Elements concentration in peat profiles approves with respect to the possibility of using trace elements concentration as indicator of the region and global environmental pollution. The $3 \mathrm{D}$ diffusion problem in $N$ layered domain described by a boundary value problem of the system of PDEs with piece-wise constant diffusion coefficients are approximate on the $2 \mathrm{D}$ boundary value problem of a system of $N$ PDEs. This algorithm is used for solving the problem of metal concentration in the 3,2 and 1 layered peat blocks. The compared numerical results with experimental data are precisely for 3-layered system.

The total advantage and attainment of an averaged method for engineering calculations is determined from the number of grid points in every of three layers. The efficiency of this method is obtained due to simple algorithms for calculations of circulant matrix. Test samples with an analytical solution (the indicator of transfer process) were developed and a numerical experiment was used to test and analyze the established method for three layers in comparison with the methods described previously. The analytical and numerical results were coincided with 3 decimal places and it means that mathematical model have a practical application in real determination of trace elements concentrations. As opposed to the models analyzed previously the newly established mathematical model envisages modeling mass transfer in N-layers, and the boundary conditions of the 3rd type included in the model enable the modeling of the substance flux through the boundary surface of the specified area in the direction of Earths interior. Mathematical modelling results and experimental data differ very slightly (completely acceptable for practice) and it means that mathematical model have practical application in real determination of trace elements concentrations. The constructed mathematical model is applicable in studying transfer processes, where substance mass is transferred through boundary surfaces of the specified area, for instance, in a purification plant, purifying works etc.

\section{References}

[1] P.A. Brown, S.A. Gill and S.J. Allen. Metal removal from wastewater using peat. Water Res., 34:3907-3916, 2000. http://dx.doi.org/10.1016/S0043-1354(00)00152-4.

[2] M. Buike and A. Buikis. System of various mathematical models for transport processes in layered strata with interlayers. WSEAS Trans. Math., 4(6):551-558, 2007.

[3] A. Buikis. Interpolation of integral average of partly-smooth function with parabolic spline. Latvian Math. Anual, 29:194-197, 1985.

[4] A. Buikis. The analysis of schemes for the modelling same processes of filtration in the underground. Riga, Acta Universitatis Latviensis, 592:25-32, 1994. (In Latvian)

[5] A. Buikis. The approximation with splines for problems in layered systems. Riga, Acta Universitatis Latviensis, 592:135-138, 1994. (In Latvian) 
[6] A. Buikis and H. Kalis. Comparison of analytical and numerical methods for simulation of radiative heating of a thin plate. Latvian J. Phys. Technical Sci., 3:20-32, 2001.

[7] E. Ideon and P. Oja. Quadratic/linear rational spline interpolation. Math. Model. Anal., 18(2):250-259, 2013. http://dx.doi.org/10.3846/13926292.2013.781071.

[8] H. Kalis. Special finite-difference approximation of flow equations in term of stream function, vorticity and velocity components for viscous incompressible liquid in curvilinear orthogonal coordinates. Comment Math. Univ. Carolinae, 34:165-174, 1993.

[9] H. Kalis. Effective finite-difference methods for the solutions of filtration problems in multilayered domains. Math. Model. Complex Anal. Proc. of the 2-nd Int. Conf., Vilnius, June 3-4, 1:84-91, 1997.

[10] G. De la Rosa, J.R. Peralta-Videa and J.L. Gardea-Torresdey. Utilization of icp/oes for the determination of trace metal binding to different humic fractions.

J. Hazardous Materials, B97:207-218, 2003. http://dx.doi.org/10.1016/S0304-3894(02)00262-5.

[11] H. Orru and M. Orru. Sources and distribution of trace elements in estonian peat. Global and Planetary Change, 53:249-258, 2006. http://dx.doi.org/10.1016/j.gloplacha.2006.03.007.

[12] J.W. Thomas. Numerical Partial Differential Equations. Finite Difference Methods. Springer-Verlag, New York, Inc., 1995.

[13] F. Zvang. Matrix Theory. Basic Results and Techniques. Springer, 1999. 